\title{
Technological proposal for cognitive modeling through the solution of problems using the game "La Escalera"
}

\author{
Author: Sneider García Holguín \\ Universidad Distrital Francisco José de Caldas \\ Bogotá, Colombia
}

\begin{abstract}
To carry out an analysis of cognitive modeling, an inclusive prototype should be used, that is, that allows the participation of the largest possible population, including the population with sensory disability, deaf or blind. In the Distrital Francisco José de Caldas University, the prototype of the game "La Escalera" is proposed, which poses a mathematical problem to be solved. This prototype consists of a board game of 8 cubes divided into two groups of 4 cubes and a board in the form of a ladder, each cube having to exchange its position with a cube of the other group respecting the rules of the game, while the game is solved, the prototype provides visual, auditory and tactile help; And in turn it collects all the movements that are made. As the user advances in the game, the data obtained from the movements made by the user with the cards will be saved in an Excel file, thus capturing the movements and errors committed by the participant.

To visualize the results obtained, a graphical interface is created in the Matlab software that allows loading the Excel file generated by the prototype. In this interface all the movements made by the user in each of the different levels of the game are observed, taking into account both the movements that lead to the solution of the game and the errors that deviate from it. The repetitive movements that the user made are also expressed in this interface and visualized with different colors for each step.
\end{abstract}

Keywords-cognitive modeling, data collection, game, learning.

\section{Introduction}

A cognitive modeling describes how thoughts on human behavior influence; this is a topic that has been developing since the twentieth century. In this modeling the perceptual and intellectual processes of the subject at the moment of solving a problem are fundamental, as well as the way in which he approaches the problem and the actions he takes to solve it.

The starting point of a cognitive modeling is perception, where the user gathers data through the senses. This is the first moment where the user interacts with the problem and generates a series of information and thoughts that he stores in his memory, as he needs this information, he will take it and use it according to his criteria.

As we move forward in this process, we find thought, in this faculty all the acquired information is processed, and links are generated between the data of this information. The reasoning, analysis, assimilation and synthesis are actions that the individual takes at this stage to solve the problem.

Language plays a very important role, since it is the tool we use to communicate with other people. The rules, rules and appropriate processes that must be taken into account when addressing the problem are obtained through language. It also allows us to acquire information that facilitates the solution of the problem, that is, there is a relationship linked to both perception and thought.

In this way, the language has to be an inclusive process, where most people can participate regardless of their disabilities. For this reason, the Distrital Francisco José de Caldas University propose the prototype of the game "La Escalera" which poses a mathematical problem to solve.

This prototype is implemented technological resources that allow integrating people with sensory disabilities, that is, people with visual and auditory impairments can interact with the prototype without any problem, thus covering a larger population group to later review the way they approach and solve a problem.

Vibration, sound and lights are some of the technological resources that were implemented in the project so that people with sensory disabilities can easily relate to the prototype and with each of the steps to follow to solve the mathematical problem.

These technological resources will be activated as the user advances in the game, thus making a feedback that will guide you to the ideal solution of the game.

Finally, all the movements made by the user are expressed in an Excel file for further analysis. This file is loaded in a graphical interface made in Matlab which allows to visualize the results obtained in the game. The numbers of movements, the repetition of steps, the trajectories or movements that the user made to arrive at the solution are some of the results captured in this interface.

\section{A. The game in education}

The game can be considered as the fundamental activity of childhood, since it is a free activity that moves away from the everyday and besides this is a great socializing instrument. 
When we perform this action we do it for pleasure and we respond to the need to have a good time without any reason.

The game is considered a methodological resource because it constitutes a basic source of learning in childhood. It focuses on enabling the global growth and development of children, providing situations that pose a challenge, but a surmountable challenge where people stand out for their ability. There is talk of a diversification of the games to avoid that the same people always stand out. This activity must be an inclusive activity, where the maximum participation of the population is generated, fostering cooperation, personal responsibility and respect for others [1].

\section{B. Sensory games}

The sensory games are mainly aimed at children with any type of sensory disability, that is, children who have a disability from one of their senses, usually referring to a visual or auditory disability.

This type of games gives children a series of benefits, and today many schools use this technique in all kinds of students [2]. Some of the benefits obtained are:

- Improvement in cognitive understanding.

- Improvement in the language.

- Reinforcement of fine motor skills in the fingers.

- Stimulation of the child's social and emotional ability.

- Recognition of feelings

- Relaxation and channeling of emotions.

- Development of imagination and creativity

\section{Cognitive model}

When solving a problem, the human being performs a series of cognitive processes that take up previously learned activities, these activities also went through a cognitive process and are now part of the subject. However, the robustness of this process varies depending on the importance and interest that we offer to the problem.

The cognitive model focuses its study on how the human being knows, thinks and remembers, analyzing the way of elaborating creating and interpreting information. Study the development of memory, thinking, intelligence, perception and strategies for solving problems

In the 1950s, a group of North American educators along with Benjamin Bloom made one of the first contributions to the education of this model. They developed the taxonomy of cognitive learning. Other authors such as David Ausubel and Jerome S. Bruner also contributed significantly to learning.

In this modeling, reference is made to the intellectual process in which emotions, thoughts and behaviors participate [3].

\section{Learning in children with sensory disabilities}

Children with sensory disabilities are those who have any type of disability in one of their senses, usually speaking of blind children and deaf children.

This type of population sharpens their other senses, allowing them to communicate with the outside world in a different but effective way.

Blind children use speech as their primary language and Braille allows them to express themselves literally with other people. At the beginning it is extremely difficult to learn this system, since your sense of touch is not very developed, but the secret is to practice and never give up [4].

People with hearing disabilities learn by sight and use sign language to communicate with other people, the support of parents and the family is essential for the child to learn, in fact some families also tend to learn the language to communicate and socialize with the child [5].

\section{E. Mathematical games}

Mathematical games usually aim to teach or practice a specific mathematical subject. Some of the subjects that are looked for to teach are the mathematical series, the classification, the numbers, the geometric figures and the measurement, among others.

The game is used as a didactic resource in the classroom. The game helps to consolidate previously learned knowledge of a topic or introduce a new topic. In addition, the use of the game develops mathematical thinking, and generates more expectation, motivation and enthusiasm in learning new concepts [6].

Some of the most used mathematical games in education are:

- The tower of Hanoi

- $\quad$ The magic square

- The jump of the frogs

- $\quad$ Sudoku

- Rubik's cube

\section{Methodology}

\section{A. Game"La Escalera"}

The prototype is based on the board game the ladder or better known as the jump of the frogs, it is a board game that consists of two fundamental parts, the board and the chips, a board in the form of a ladder is proposed, and the chips will be 8 cubes divided into two groups 4 red cubes and 4 blue cubes. 


\section{B. Structure}

The basic structure of the prototype consists of a staircase consisting of 9 steps, the first four steps are located ascending, while the last 4 steps are descending, in the fifth step there is a break that the structure is shown in the Figure 1.

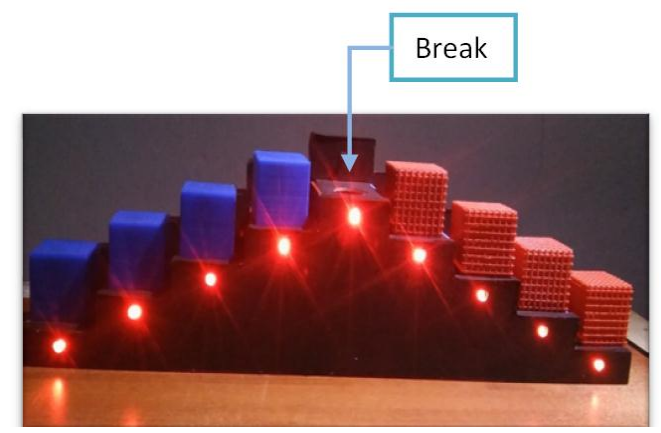

Figure 1 Structure of the game "La Escalera"

Managing the structure by steps provides easy positioning when placing the chips (cubes) of the game, this was especially done to help blind people to provide more precise movements with the chips.

Each step has a Radio Frequency Identification Module (RFID), at the bottom of each step there is the visual support (LEDs) and on a base perpendicular to the structure, infrared light emitting diodes are located to activate the vibration of the cards when an erroneous movement occurs, it is good to clarify that within the structure are components and electronic circuits that make possible the control and decision making of the prototype.

\section{Game Chips}

Managing the structure by steps provides easy positioning when placing the chips (cubes) of the game, this was especially done to help blind people to provide more precise movements with the chips.

The chips of the game are 8 cubes, divided into two groups, the first group is 4 cubes of blue color and the second group are 4 cubes of red color, the cubes have dimensions of $45 \times 45 \times 45 \mathrm{~mm}$ and each group of cubes has a texture that differentiates them from the other group, this texture was made on a 3D printer. The red cubes have a raised grid, while the blue cubes are completely smooth (see Figure 2); this is done so that blind people differentiate the color or the group to which each cube belongs only by touching them. In addition, on one side of the cubes is a hole with a diameter of approximately $5 \mathrm{~mm}$, where an infrared receiver is positioned (see Figure 3) and in the lower face of the cube there is another hole with a diameter of $25 \mathrm{~mm}$ where a passive RFID Chip fits (see Figure 3) that allows to position the cubes in the software. Finally, in its interior there is an electronic circuit that allows vibrating movements with the cube, the circuit works like a switch, if the receiver receives an infrared light the cubes vibrate, otherwise the cubes will remain at rest.

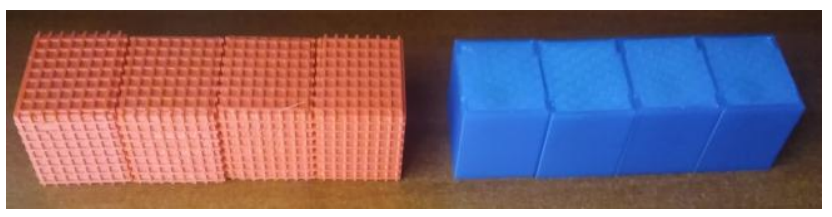

Figure 2 Game Cubes

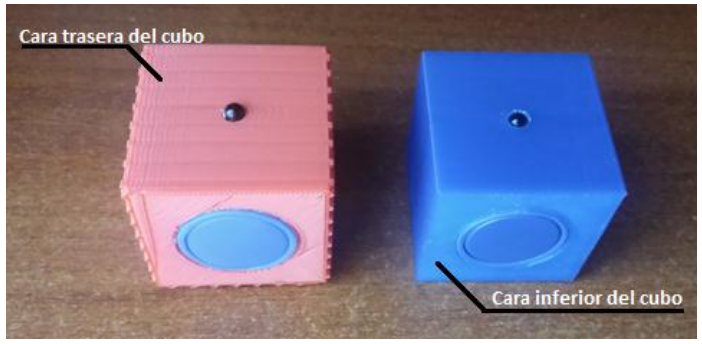

Figure 3 Location of the sensors in each of the cubes

\section{Explanation of the game}

As previously stated, the game has 8 cubes, 4 blue and 4 red. The red cubes are organized on one side of the ladder and the blue cubes on the opposite side in such a way that each step has a cube, except for the fifth step or landing which must be free to start the game as shown in Figure 1.

Start the game when the cubes are organized, the main objective of the game is to exchange the position of the red cubes with the position of the blue cubes, that is, move both the red cubes and the blue cubes to their opposite side. To achieve this goal, the following basic rules of the game must be met:

1. The cubes can only be moved in the direction of their opposite color.

2. Each cube can be moved to the next box if it is free.

3. The cubes can jump on a cube of opposite color if the next box is empty.

If you get to break any of these rules or you are in a time where the game cannot advance, is the movement does not lead me to the solution, the user will have to start the game again.

Helps:

When the person is trying to solve the game, the prototype has systems that warn the user if he has made an error or if he is taking a path that does not lead to the solution of the problem. It should be noted that these systems can be activated and deactivated at any time during the game, although for the blind and deaf blind people they will have their default settings. The systems are the following:

- Light system

- $\quad$ Sound system

- Vibration system

Afterwards, the operation of each one will be explained. 


\section{E. Auditory, visual and tactile feedback.}

The development of the tests is carried out with 11 users with an age range of 4-30 years, who do not present any disability; the game is presented: the structure, chips and the rules of the game are explained. Several present confusions with the rules of the game, so it has to be explained more than once. Before starting the game, the camera is positioned in such a way that it captures the user's face as much as possible in order to have reliable data, for this reason it is also important to take into account the lighting of the face and diligence on the user's basic information.

Internally the structure has a micro-controller, which processes the data acquired from the movements made by the user with the cubes and their mistakes, to later make decisions and execute a specific task, generate a sound, turn on the lights (LEDs) or vibrate the cubes.

Mainly there are 3 important stages of the game where feedback is a fundamental part.

The first stage is the calibration system, this stage is the most important part of the game, because it is where the tools that provide visual, auditory or tactile support are activated or deactivated, these can be activated or deactivated during the course of the game. This stage is also very important because the user's first interaction with the game is performed, in this stage the user must place the cubes on the board and the prototype will communicate in different ways with the user indicating if the positions of the cubes are the suitable to start the game.

Below is a more detailed explanation of each of the systems and emphasis is on what moments will be activated.

\section{1) Sound}

This system generates different tones for each step, as the user makes a movement, the software generates a code that will allow an audio corresponding to the musical tones Do, Do \#, Re, Re \#, Mi, Fa, Sol, La, Si, respectively for each step.

In addition to this, this system also performs other tasks that mainly provide blind people with extra help to locate themselves in the game:

- At the beginning of the game when the boxes are placed, initially there is a calibration system, this system allows the user to indicate if the position where the box is located is appropriate to start the game, the user has two possibilities, right or left of the step-landing step figure 1 , if the position is incorrect a characteristic sound is generated indicating that an error has been made, and you will have to place the cube in its opposite orientation. Once the previous calibration is done, the prototype tells the user that the game can begin.

- In the course of the game, the prototype indicates that the cube was placed correctly in the desired step, if the movement is valid, the musical tone assigned to the step where the cube is positioned is generated, if the movement is incorrect, it indicates the user through a characteristic sound that its movement is incorrect and the game has no solution, in this case the user will have to start the game again.

- When the game is made by levels, it is announced at what level the game will develop and at the moment of completing this level a sound is provided that emphasizes its triumph.

- When the game is completed with the complete tiles (8 cubes), it indicates that the game is finished completely.

\section{2) Lights}

The lighting system provides 6 visual indications by RGB Light Emitting Diodes (LEDs) which are:

- At the start of the program in the calibration stage, it is indicated by the LEDs if the cube is in the proper position to start the game, if so it will turn green, otherwise it will emit a red color.

- When the cubes are placed in the correct position, all the green LEDs will light up and flash for approximately 1 second.

- When a movement is generated, 2 yellow LEDs come on, one indicating the previous position and the other the new position of the cube. It should be noted that the 2 LEDs correspond to the steps involved with the last two movements.

- If a rule is broken or the user tries to go by a path that does not reach the solution, the red LEDs turn on to indicate that there is an error and the user has to start the game again or the level at which it's found.

- When one of the levels is correctly completed, the LEDs will flash and change their color emitted for an established time.

- At the end of the game, the LEDs will flash again and change their color but without any time limit.

This system was designed primarily for deaf people, so they can more easily identify the state of the game.

\section{3) Vibration}

For the vibration system is implemented a wireless circuit with infrared light emitting diodes and infrared receivers, as there are no cables, the user plays comfortably and his movements are not hindered by external elements.

The vibration of the cubes is given thanks to an electronic circuit that is inside the cubes, this electronic circuit drives a vibrating motor depending on an input signal, this vibrator motor is attached to the top or top face of the cube and At the moment of activating it vibrates the entire cube. The input signal that makes possible the vibration of the cubes is an infrared light emitted by the infrared LEDs that are in the base perpendicular to the structure. These LEDs will emit an infrared light when they are indicated, if the receiver that is in the cube perceives this infrared light, the internal circuit is 
activated and the vibrating motor vibrates the cube with which the last movement was made.

The tasks performed by this system are the following:

- To start the game, the cubes must be placed correctly, the red cubes must be on the left side of the ladder and the blue cubes on the right side, if this is not so, the cube that is in the wrong position it will vibrate.

- Indicate when one of the rules has been broken.

- Indicate when the game no longer leads to a solution.

In case 2 and 3, the user must start the game again, since this error will not allow him to continue advancing in the solution of the mathematical problem.

In case the game is going to be used by a deaf-blind person, a series of predetermined parameters will be activated that will allow the operation of a system of vibratory pulses that will help this population to understand each stage of the game, whether the beginning of the game, the correct and incorrect movements and the end of the game.

\section{F. Graphic interface}

Through the integrated development environment provided by Microsoft Visual Studio, a graphical interface is made that allows the control of the different activities that the prototype develops. By default, the game starts at level 4, but if the user wants to reduce the level of complexity of the game it can be done by selecting any other level that the interface provides. In the user information we provide the disability and the name of the player, this name will also be carried by the Excel file that is generated. The default feedback systems are activated, but these can be disabled at the start or end of the game.

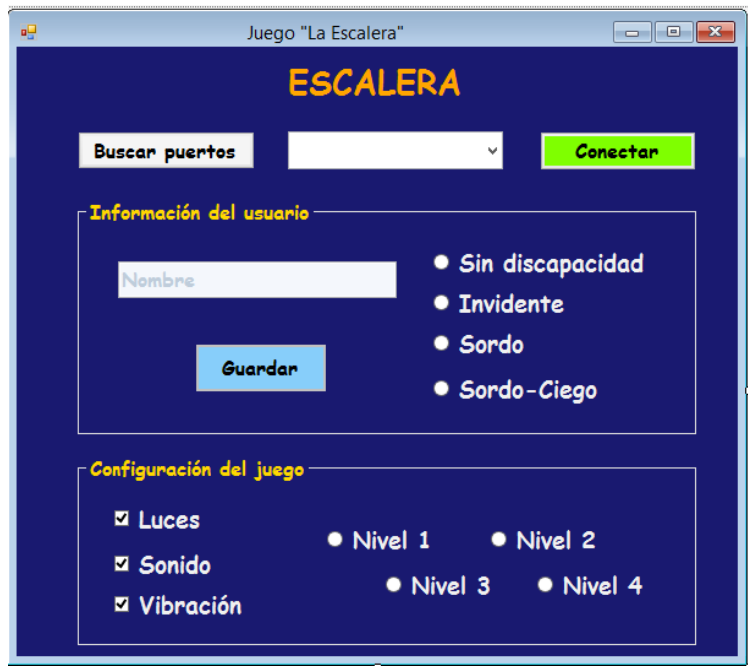

Figure 4 Graphical interface in Visual Studio

\section{Results}

15 people interact with the game, the prototype's feedback systems comply with the proposed functions, and in addition all the movements made by the users with the chips of the game are saved and captured in an Excel file as shown in figure 5 .

\begin{tabular}{|c|c|c|c|c|c|c|c|c|c|c|c|c|c|c|}
\hline$\Delta$ & A & & B & $c$ & & D & E & & $\mathrm{F}$ & G & & $\mathrm{H}$ & I & \\
\hline \multicolumn{15}{|c|}{ ion 3 Posicion 4 Posicion 5 Posicion 6 Posicion 7 Posicion 8 Posicion 9} \\
\hline 54 & & 1 & & 2 & 3 & 0 & & 5 & 4 & 4 & 6 & & 7 & 8 \\
\hline 55 & & 1 & & 2 & 0 & 3 & & 5 & 4 & 4 & 6 & & 7 & 8 \\
\hline 56 & & 1 & & 2 & 5 & 3 & & 0 & 4 & 4 & 6 & & 7 & 8 \\
\hline 57 & & 1 & & 2 & 5 & 3 & & 6 & 4 & 4 & 0 & & 7 & 8 \\
\hline 58 & & 1 & & 2 & 5 & 3 & & 6 & 4 & 4 & 7 & & 0 & 8 \\
\hline 59 & & 1 & & 2 & 5 & 3 & & 6 & 0 & 0 & 7 & & 4 & 8 \\
\hline 60 & & 1 & & 2 & 5 & 0 & & 6 & 3 & 3 & 7 & & 4 & 8 \\
\hline 61 & & 1 & & 0 & 5 & 2 & & 6 & 3 & 3 & 7 & & 4 & 8 \\
\hline 62 & & 0 & & 1 & 5 & 2 & & 6 & 3 & 3 & 7 & & 4 & 8 \\
\hline 63 & & 5 & & 1 & 0 & 2 & & 6 & 3 & 3 & 7 & & 4 & 8 \\
\hline 64 & & 5 & & 1 & 6 & 2 & 2 & 0 & 3 & 3 & 7 & & 4 & 8 \\
\hline 65 & & 5 & & 1 & 6 & 2 & 2 & 7 & 3 & 3 & 0 & & 4 & 8 \\
\hline 66 & & 5 & & 1 & 6 & 2 & 2 & 7 & 3 & 3 & 8 & & 4 & 0 \\
\hline 67 & & 5 & & 1 & 6 & 2 & 2 & 7 & 3 & 3 & 8 & & 0 & 4 \\
\hline 68 & & 5 & & 1 & 6 & 2 & 2 & 7 & 0 & 0 & 8 & & 3 & 4 \\
\hline 69 & & 5 & & 1 & 6 & 0 & 0 & 7 & 2 & 2 & 8 & & 3 & 4 \\
\hline 70 & & 5 & & 0 & 6 & 1 & 1 & 7 & 2 & 2 & 8 & & 3 & 4 \\
\hline 71 & & 5 & & 6 & 0 & 1 & 1 & 7 & 2 & 2 & 8 & & 3 & 4 \\
\hline 72 & & 5 & & 6 & 7 & 1 & 1 & 0 & 2 & 2 & 8 & & 3 & 4 \\
\hline 73 & & 5 & & 6 & 7 & 1 & 1 & 8 & 2 & 2 & 0 & & 3 & 4 \\
\hline 74 & & 5 & & 6 & 7 & 1 & 1 & 8 & 0 & 0 & 2 & & 3 & 4 \\
\hline 75 & & 5 & & 6 & 7 & 0 & 0 & 8 & 1 & 1 & 2 & & 3 & 4 \\
\hline 76 & & 5 & & 6 & 7 & 8 & 8 & 0 & 1 & 1 & 2 & & 3 & 4 \\
\hline
\end{tabular}

Figure 5 Excel document generated

To clearly observe the results obtained in each level of the game, the Excel file is loaded into a graphical interface made in Matlab, which allows to observe the amount of movements made, and the colors of the repetition of steps or moves made by the user are differentiated by colors like the trajectories or moves that the user chose to solve the game. See figure 6 .

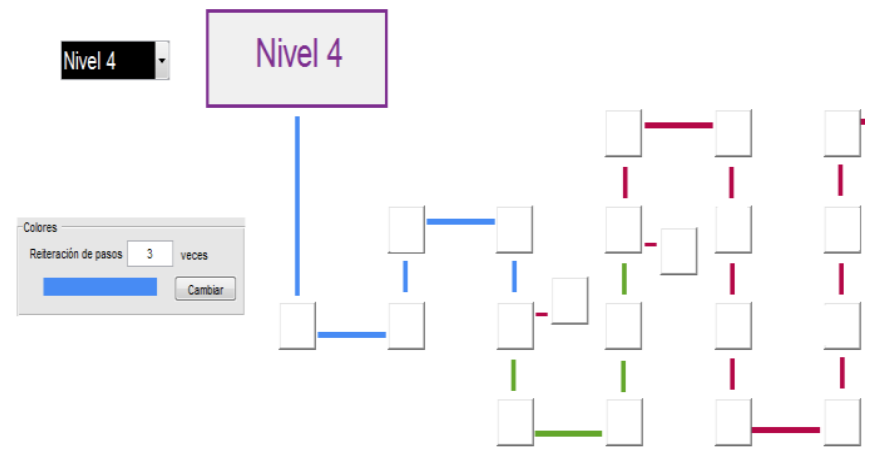

Figure 6 information display in Matlab 


\section{IV.}

\section{v. Conclusions}

All the movements that are made with the chips inside the board are saved successfully and sent to the micro-controller to be subsequently converted into an Excel File.

The feedback systems worked correctly and told the user the steps taken with the cards, their mistakes and the achievements.

Blind people easily recognize and differentiate red cubes and blue cubes; this was possible thanks to the fact that a different texture was handled for each color.

Some users do not end the game because of frustration, grief or boredom, but still keep the movements and plays made by them in an Excel file, and if we load this file in the graphical interface made in Matlab webcam see the plays and repetitive steps that they committed.

The majority of the evaluated population starts the game at the most complex level, and some of them, unable to complete the game, decides to reduce the level and start with the first level, the other part of the participants decides to continue at the same level until completing it or surrender.

\section{References}

[1] R. Montealegre, "La solución de problemas cognitivos en estudiantes de psicología,” Acta Colomb. Psicol, pp. 119-138, 2011.

[2] “Juegos sensoriales para niños - Ejemplos, juguetes y clases," [En línea]. Disponible en: http://lahabitaciondemipeque.com/juegossensoriales-para-ninos-primaria-ejemplos-beneficios/. [Consultado: 27ago-2018].

[3] "El modelo cognitivo - El modelo cognitivo," [En línea]. Disponible en: https://sites.google.com/site/elmodelocognitivo/el-modelo-cognitivo. [Consultado: 27-ago-2018].

[4] P. M. Andrade, "Alumnos con discapacidad visual Necesidades y respuesta educativa," Educacion.Once.Es, pp. 1-75, 2010.

[5] R. Montealegre, «La solución de problemas cognitivos en estudiantes de psicología», Acta Colomb. Psicol., pp. 119-138, 2011.

[6] M.Alibali, R.Spencer, L.Knox, y S.Kita, "Spontaneous gestures influences strategy choices in problem solving," Psychol. Sci., pp. 11381144, 2011. 